\title{
Novel multifunctional stimuli-responsive nanoparticles for synergetic chemo-photothermal therapy of tumors
}

Xing-Qun Pu, Xiao-Jie Ju, ${ }^{,}{ }_{,+}^{\dagger}$ Lei Zhang, ${ }^{\dagger}$ Quan-Wei Cai, ${ }^{\dagger}$ Yu-Qiong Liu, ${ }^{\dagger}$ Han-Yu Peng, ${ }^{\dagger}$ Rui

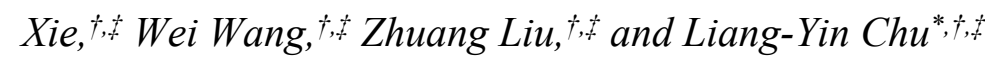

${ }^{\dagger}$ School of Chemical Engineering, Sichuan University, Chengdu, Sichuan 610065, China

†State Key Laboratory of Polymer Materials Engineering, Sichuan University, Chengdu,

Sichuan 610065, China

Corresponding Authors

*E-mail: chuly@scu.edu.cn (L.-Y.C.)

*E-mail: juxiaojie@scu.edu.cn (X.-J. J.)

\section{Content}

Supplementary Figures S1-S13

Supplementary Tables S1-S3 


\section{Supplementary Figures S1-S13}

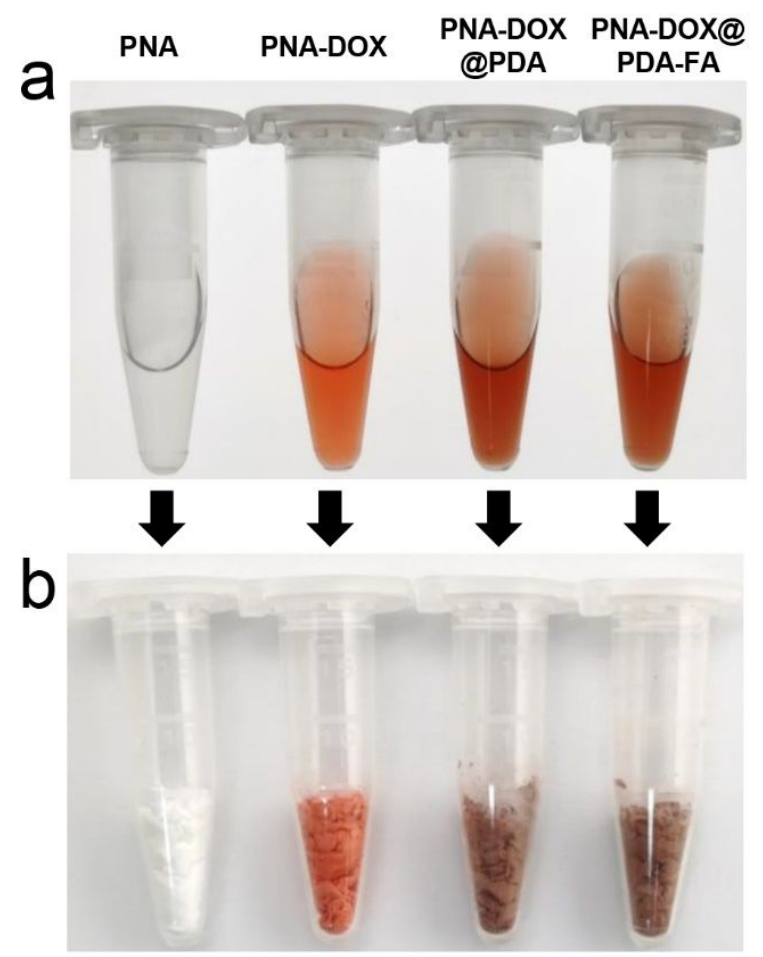

Figure S1. The optical photographs of the nanoparticle suspensions with concentration of $0.5 \mathrm{mg} \mathrm{mL}^{-1}$ (a) and the freeze-dried nanoparticles (b). 

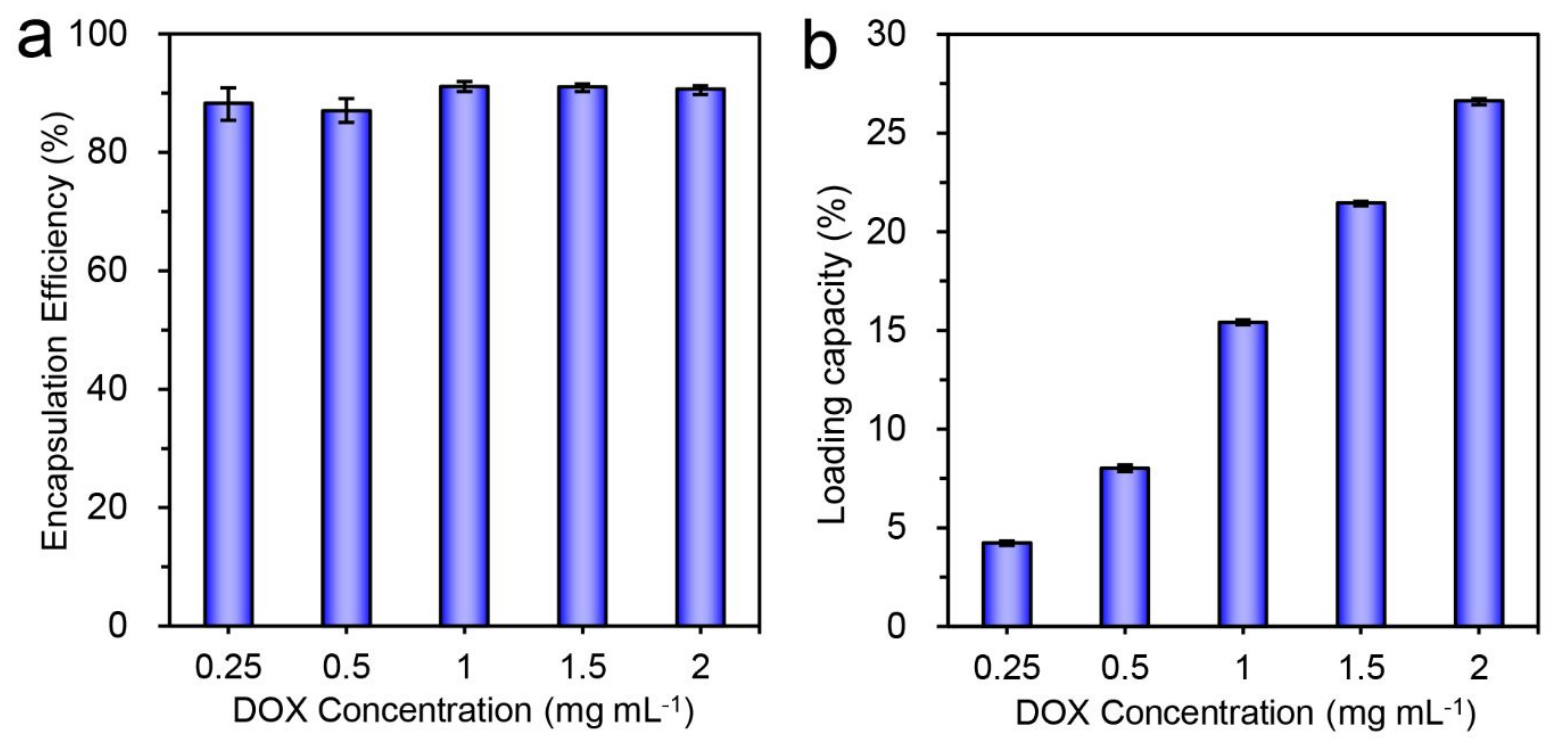

Figure S2. The drug encapsulation efficiency (a) and loading capacity (b) of PNA-DOX nanoparticles with different concentrations of DOX. 

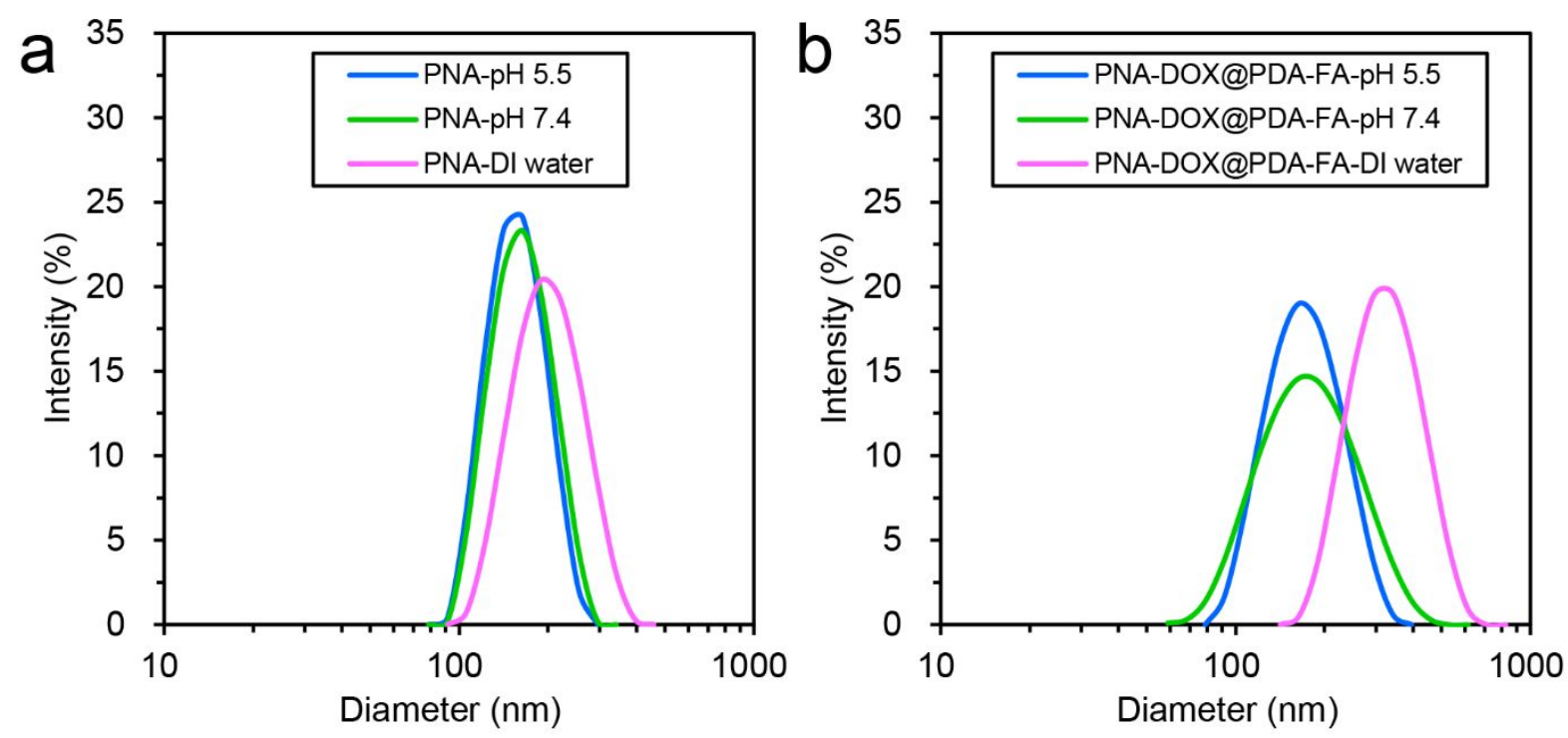

Figure S3. Hydrodynamic diameters distributions of PNA (a) and PNA-DOX@PDA-FA (b) nanoparticles dispersed in PBS buffers ( $\mathrm{pH} 5.5$ and $\mathrm{pH} 7.4)$ and DI water at $25^{\circ} \mathrm{C}$. 

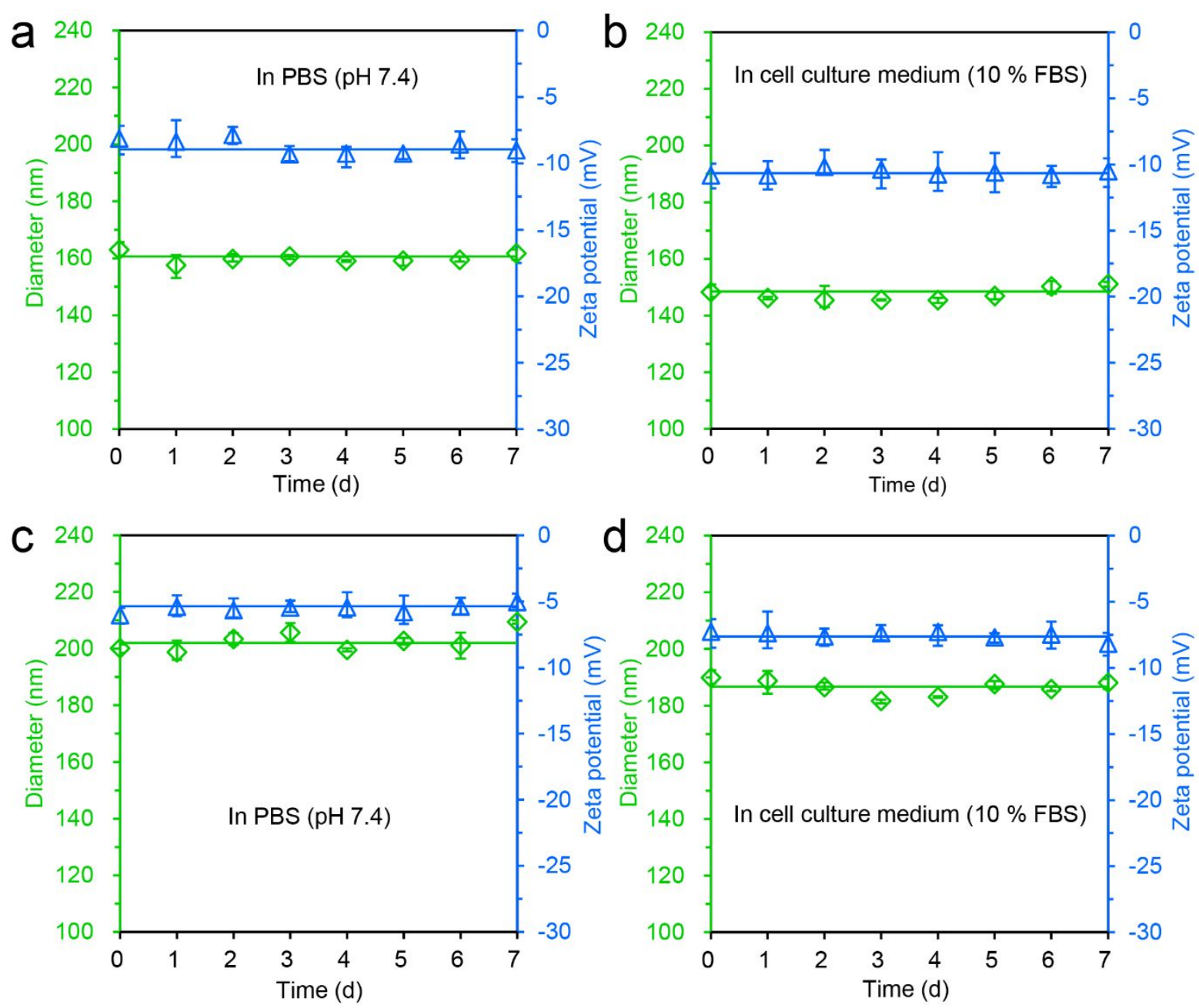

Figure S4. The size and zeta potential changes of PNA $(a, b)$ and PNA-DOX@PDA-FA (c, d) nanoparticles incubated in PBS (pH 7.4) (a, c) and cell culture medium (RAPI 1640, $10 \%$ FBS) (b, d) for $7 \mathrm{~d}$. 


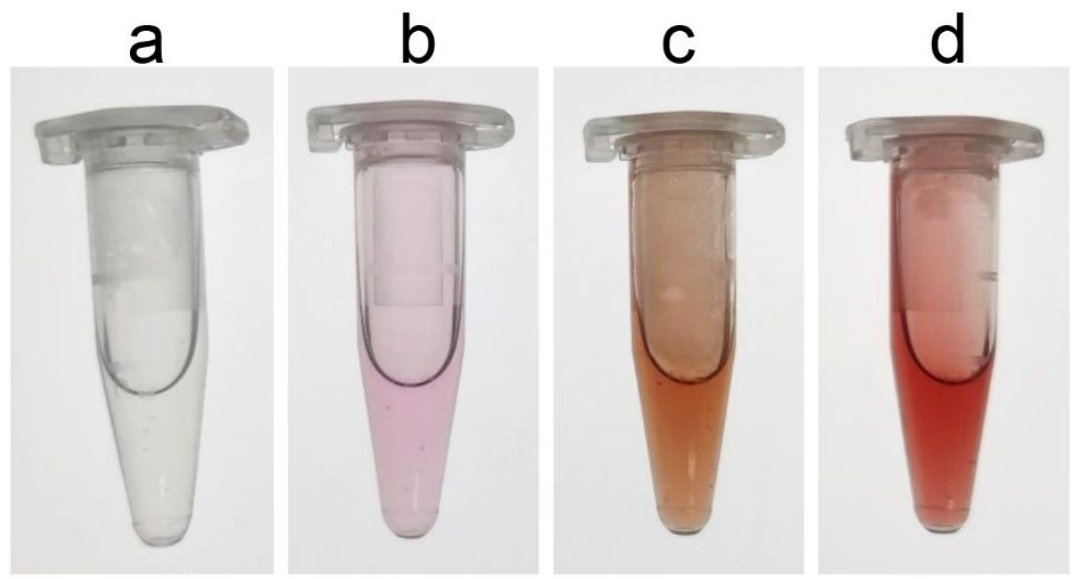

Figure S5. The optical photographs of PNA (a, b) and PNA-DOX@PDA-FA (c, d) nanoparticles incubated in PBS ( $\mathrm{pH} 7.4$ ) (a, c) and cell culture medium (RAPI 1640, 10 \% FBS) $(b, d)$ after $7 d$. 

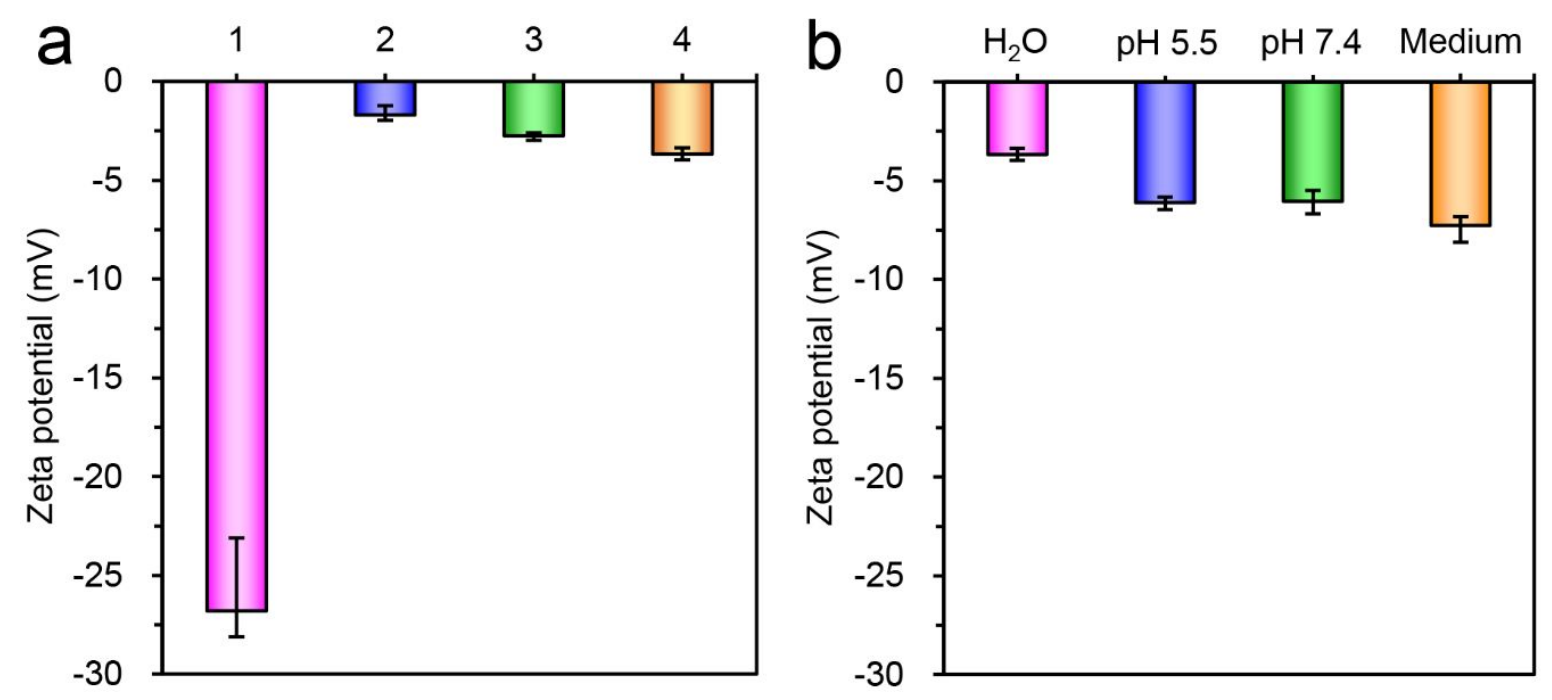

Figure S6. (a) The zeta potential values of PNA (1), PNA-DOX (2), PNA-DOX@PDA (3) and PNA-DOX@PDA-FA (4) nanoparticles in DI water. (b) The zeta potential values of PNA-DOX@PDA-FA nanoparticles in DI water, pH 7.4 PBS, pH 5.5 PBS and cell culture medium (10\% FBS). 


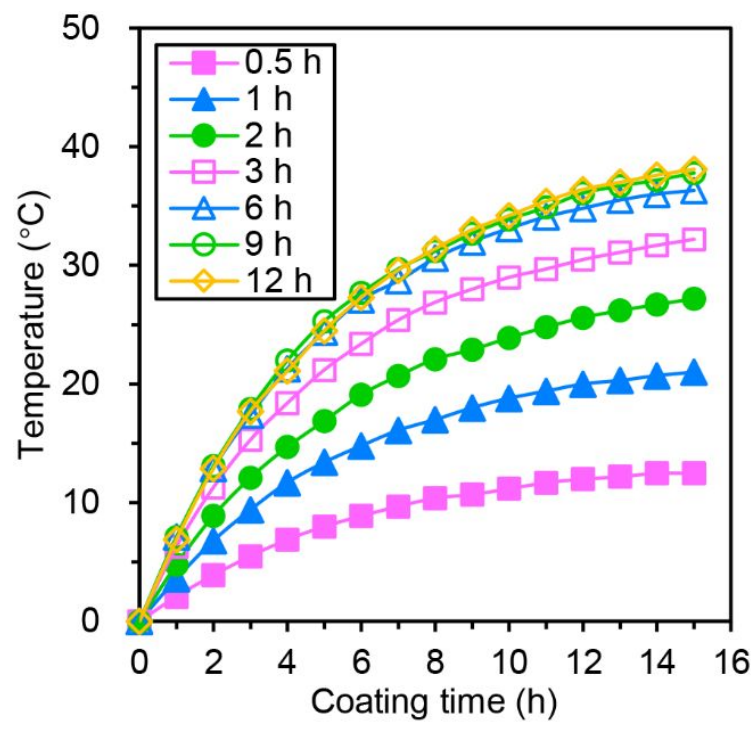

Figure S7. Photothermal heating curves of PNA-DOX@PDA nanoparticles $\left(0.5 \mathrm{mg} \mathrm{mL}^{-1}\right)$ with different PDA coating time periods under NIR laser irradiation $\left(808 \mathrm{~nm}, 2 \mathrm{~W} \mathrm{~cm}^{-2}\right)$ for 15 $\min$. 

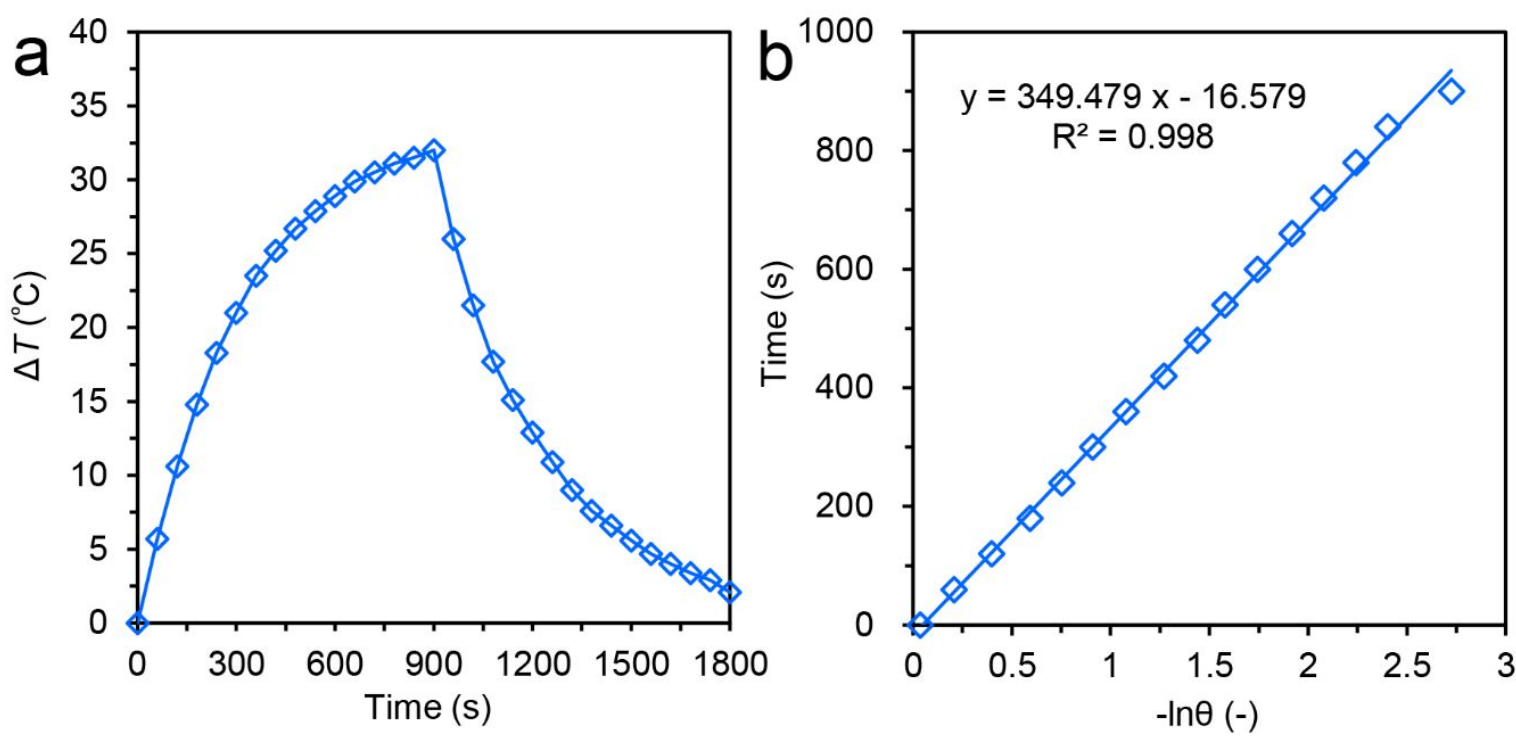

Figure S8. (a) The photothermal heating curve of the PNA-DOX@PDA-FA nanoparticle solution $\left(0.5 \mathrm{mg} \mathrm{mL}^{-1}\right)$ under one on/off cycle of NIR laser irradiation $\left(808 \mathrm{~nm}, 1 \mathrm{~W} \mathrm{~cm}^{-2}\right)$. The NIR laser is turned on from 0 to $900 \mathrm{~s}$, and then is turned off from 900 to $1800 \mathrm{~s}$. (b) The linear fitting curve of cooling time versus negative natural logarithm of driving force temperature, which is obtained from the cooling stage of Figure S6a. 


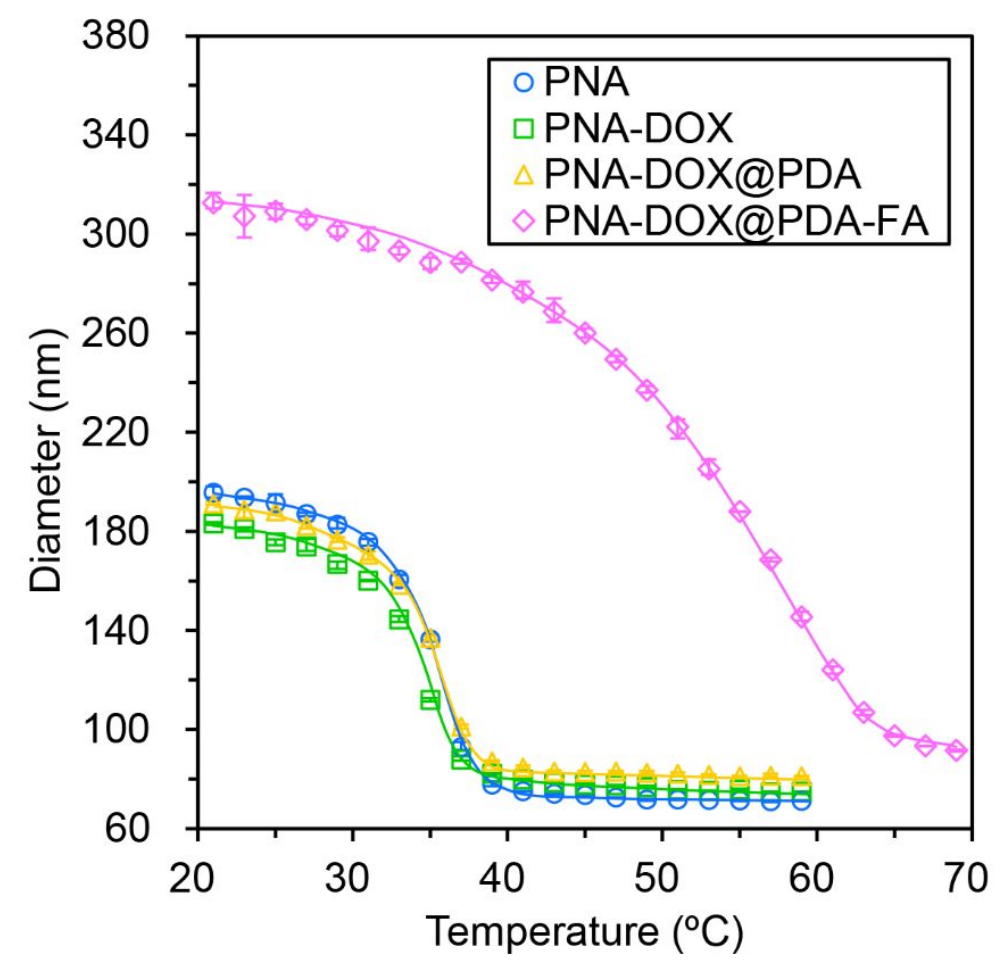

Figure S9. Hydrodynamic diameters of PNA, PNA-DOX, PNA-DOX@PDA and PNADOX@PDA-FA nanoparticles in DI water as a function of temperature. 

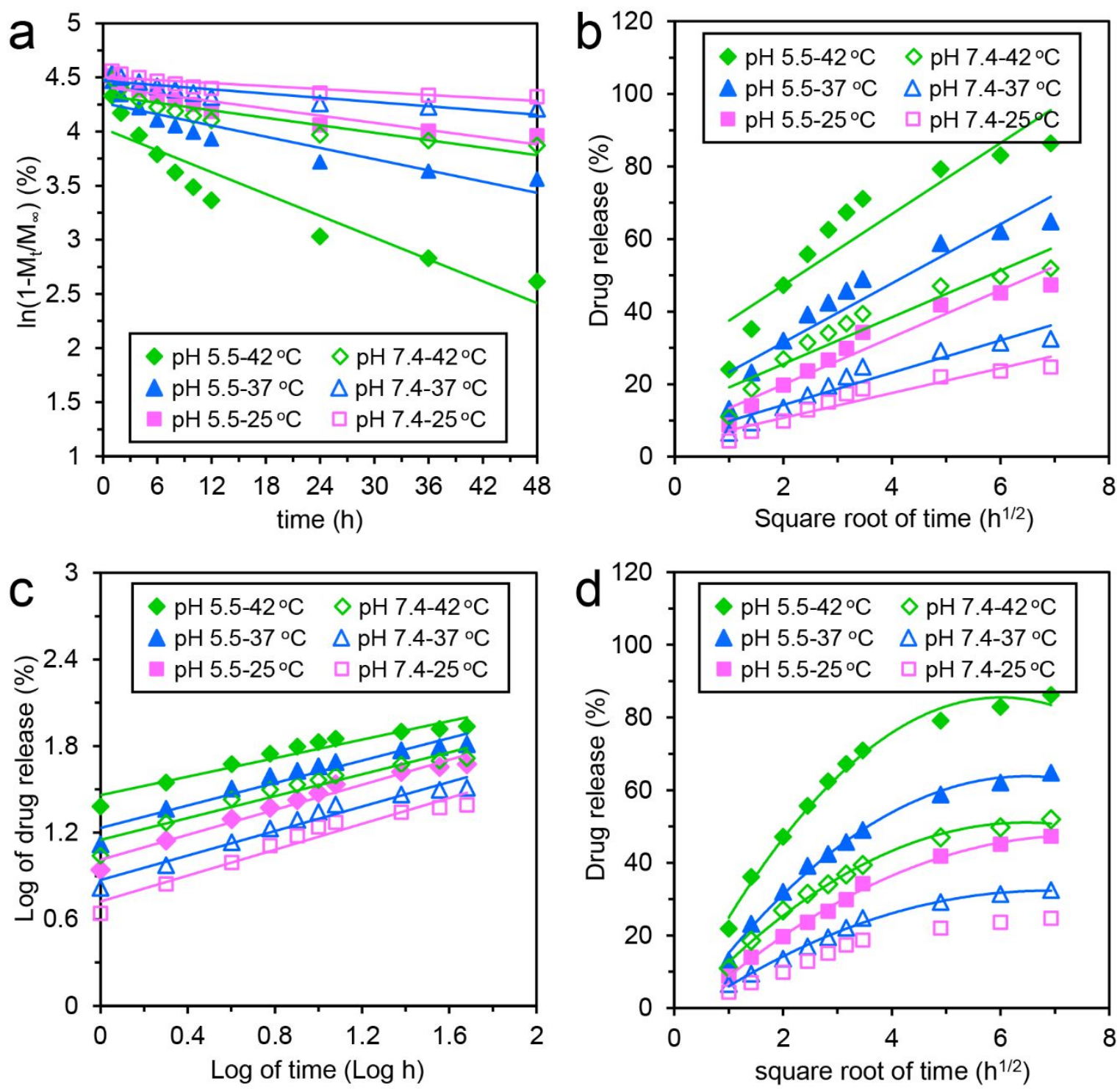

Figure S10. The drug release profiles of PNA-DOX nanoparticles fitted in first-order (a), Higuchi (b), Korsmeyer-Peppas (c) and Peppas-Sahlin (d) models. 

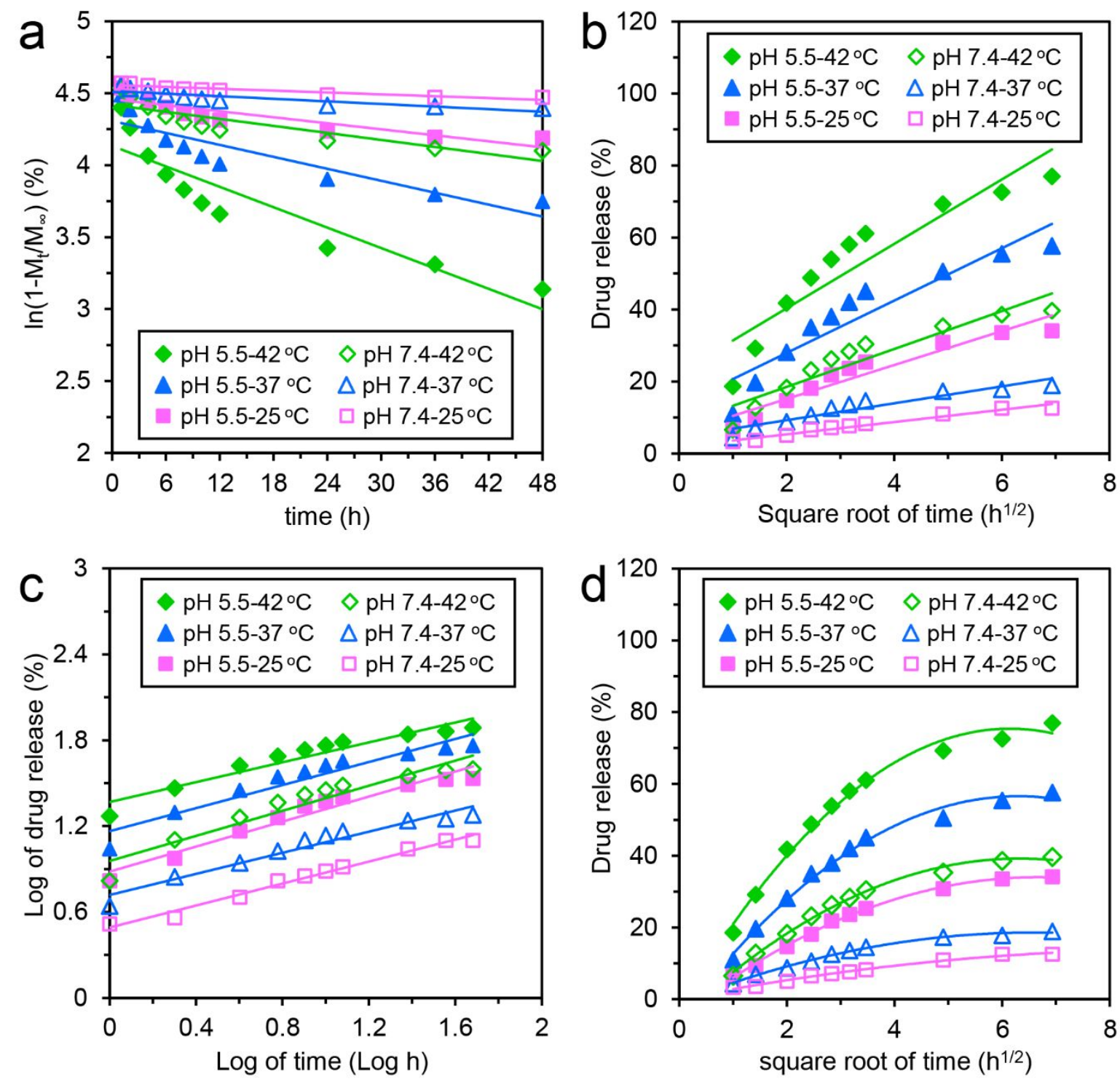

Figure S11. The drug release profiles of PNA-DOX@PDA nanoparticles fitted in first-order (a), Higuchi (b), Korsmeyer-Peppas (c) and Peppas-Sahlin (d) models. 

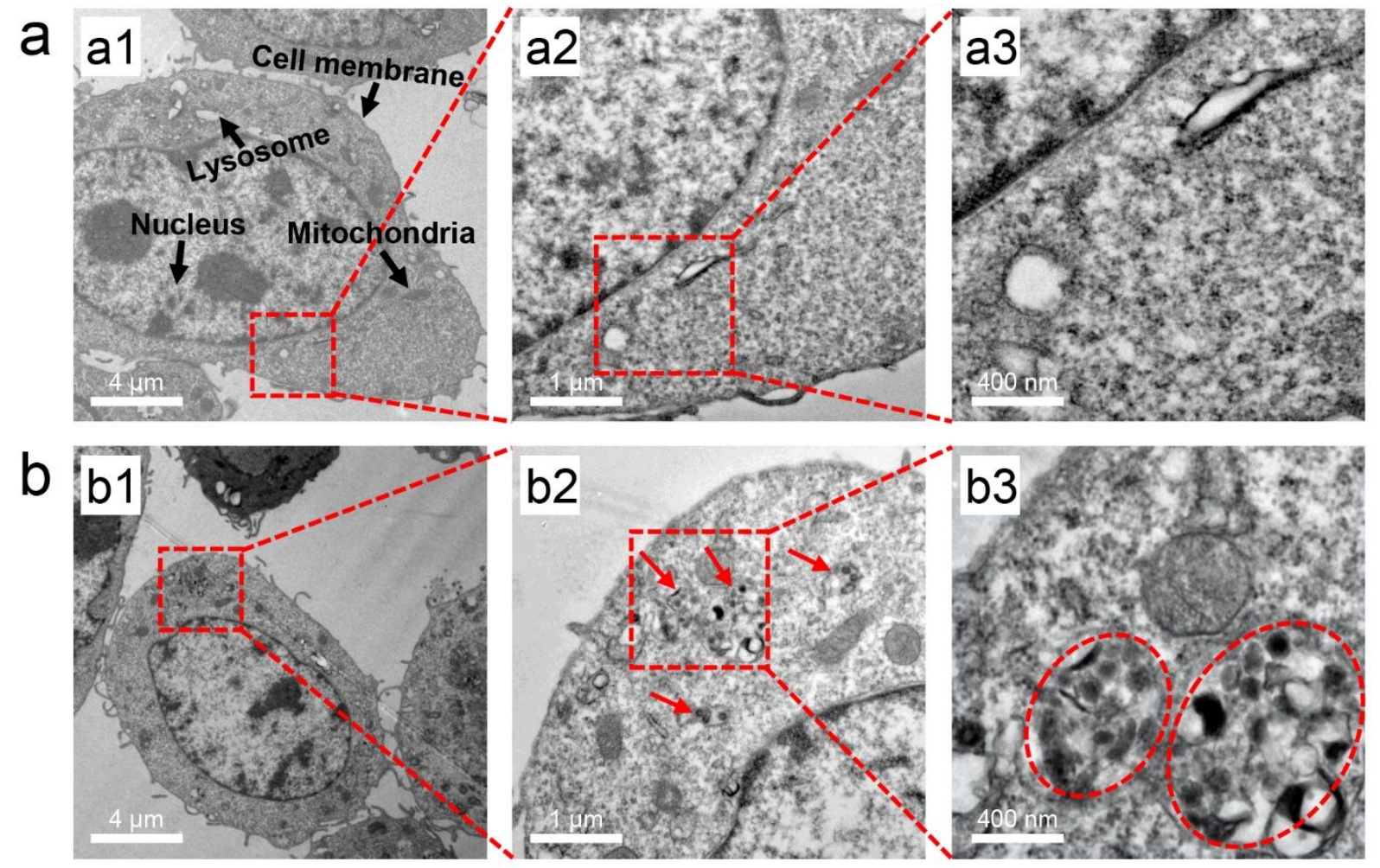

Figure S12. TEM images of 4T1 cells after incubation with PBS (a) and PNA-DOX@PDAFA nanoparticles $\left(200 \mu \mathrm{g} \mathrm{mL}^{-1}\right)(\mathrm{b})$ for $12 \mathrm{~h}$. 

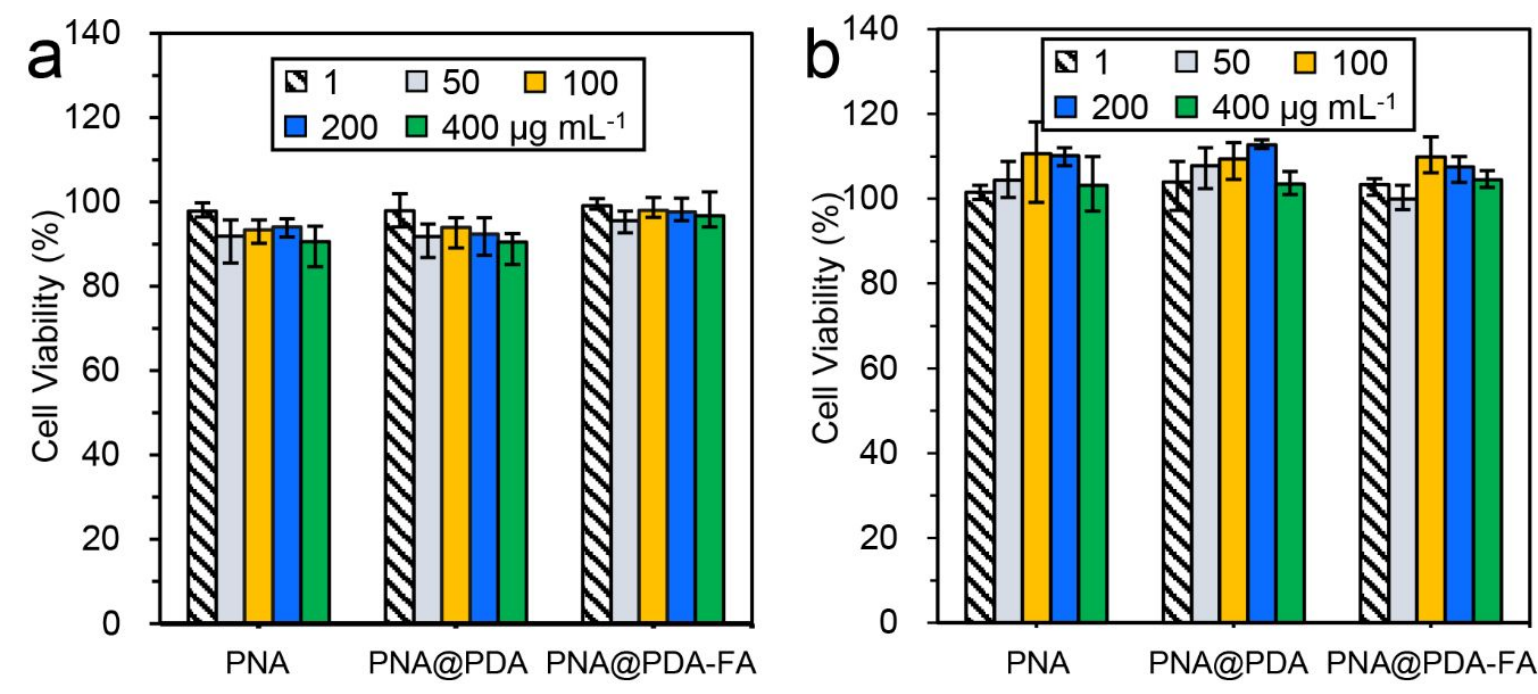

Figure S13. The cell viability of Hela (a) and L929 (b) cells after incubation with various concentrations of PNA@PDA-FA nanoparticles for 24 h. 


\section{Supplementary Tables S1-S3}

Table S1. Element analysis results of various nanoparticles

\begin{tabular}{cccc}
\hline \multirow{2}{*}{ Sample } & \multicolumn{3}{c}{ Relative content of elements (\%) } \\
\cline { 2 - 4 } & $\mathrm{C}$ & $\mathrm{N}$ & $\mathrm{O}$ \\
\hline PNA & 75.37 & 9.61 & 15.02 \\
PNA-DOX & 75.25 & 7.42 & 17.33 \\
PNA-DOX@PDA & 75.07 & 8.33 & 16.60 \\
PNA-DOX@PDA-FA & 75.76 & 2.32 & 21.92 \\
\hline
\end{tabular}


Table S2. Release kinetics parameters of PNA-DOX and PNA-DOX@PDA nanoparticles fitted in first-order, Higuchi, Korsmeyer-Peppas and Peppas-Sahlin kinetic models

\begin{tabular}{|c|c|c|c|c|c|c|c|c|c|c|c|c|}
\hline \multirow{2}{*}{ Sample } & \multirow{2}{*}{$\mathrm{pH}$} & \multirow{2}{*}{$\mathrm{T}\left({ }^{\circ} \mathrm{C}\right)$} & \multicolumn{2}{|c|}{ First-order } & \multicolumn{2}{|c|}{ Higuchi } & \multicolumn{3}{|c|}{ Korsmeyer-Peppas } & \multicolumn{3}{|c|}{ Peppas-Sahlin } \\
\hline & & & $k_{1}$ & $R^{2}$ & $k_{2}$ & $R^{2}$ & $k_{3}$ & $n$ & $R^{2}$ & $k_{4}$ & $k_{5}$ & $R^{2}$ \\
\hline \multirow{6}{*}{$\begin{array}{l}\text { PNA- } \\
\text { DOX }\end{array}$} & \multirow{3}{*}{5.5} & 25 & 0.011 & 0.865 & 6.509 & 0.943 & 10.290 & 0.433 & 0.968 & 12.919 & -0.934 & 0.997 \\
\hline & & 37 & 0.017 & 0.843 & 8.156 & 0.895 & 17.077 & 0.389 & 0.927 & 21.030 & -1.623 & 0.995 \\
\hline & & 42 & 0.034 & 0.875 & 9.783 & 0.855 & 28.794 & 0.322 & 0.930 & 29.057 & -2.419 & 0.989 \\
\hline & \multirow{3}{*}{7.4} & 25 & 0.005 & 0.781 & 3.407 & 0.906 & 5.282 & 0.448 & 0.947 & 8.453 & -0.636 & 0.994 \\
\hline & & 37 & 0.007 & 0.807 & 4.434 & 0.918 & 7.435 & 0.424 & 0.961 & 10.542 & -0.770 & 0.996 \\
\hline & & 42 & 0.012 & 0.812 & 6.437 & 0.893 & 14.096 & 0.379 & 0.927 & 16.642 & -1.287 & 0.994 \\
\hline \multirow{6}{*}{$\begin{array}{l}\text { PNA- } \\
\text { DOX } \\
\text { @PDA }\end{array}$} & \multirow{3}{*}{5.5} & 25 & 0.007 & 0.799 & 4.706 & 0.910 & 7.630 & 0.437 & 0.951 & 11.613 & -0.871 & 0.998 \\
\hline & & 37 & 0.014 & 0.807 & 7.288 & 0.877 & 14.615 & 0.403 & 0.913 & 19.671 & -1.561 & 0.990 \\
\hline & & 42 & 0.024 & 0.845 & 8.960 & 0.861 & 23.356 & 0.348 & 0.919 & 25.293 & -2.060 & 0.989 \\
\hline & \multirow{3}{*}{7.4} & 25 & 0.002 & 0.875 & 1.704 & 0.968 & 3.102 & 0.384 & 0.986 & 2.954 & -0.158 & 0.991 \\
\hline & & 37 & 0.003 & 0.766 & 2.352 & 0.900 & 5.234 & 0.369 & 0.949 & 5.943 & -0.453 & 0.994 \\
\hline & & 42 & 0.008 & 0.776 & 5.265 & 0.883 & 9.066 & 0.437 & 0.911 & 14.092 & -1.113 & 0.994 \\
\hline
\end{tabular}


Table S3. $\mathrm{IC}_{50}$ value on $4 \mathrm{~T} 1$ cells after incubated with free DOX and DOX-loaded nanoparticles for $24 \mathrm{~h}$ and $48 \mathrm{~h}$.

\begin{tabular}{cccccc}
\hline \multirow{2}{*}{$\begin{array}{c}\text { Incubation } \\
\text { Time (h) }\end{array}$} & DOX & PNA-DOX & $\begin{array}{c}\text { PNA-DOX } \\
\text { @PDA }\end{array}$ & $\begin{array}{c}\text { PNA-DOX } \\
\text { @PDA-FA }\end{array}$ & $\begin{array}{c}\text { PNA-DOX@PDA- } \\
\text { FA+NIR }\end{array}$ \\
\hline 24 & 0.49 & 5.69 & 15.33 & 7.20 & 3.70 \\
48 & 0.16 & 2.08 & 3.46 & 1.59 & 1.02 \\
\hline
\end{tabular}

